\title{
Melanoma: a new landmark in targeted oncology
}

\author{
Jean-François Morère
}

Received: 4 August 2011 /Accepted: 4 August 2011 /Published online: 20 August 2011

(C) Springer-Verlag 2011

The landscape of metastatic melanoma has been particularly dull for decades. The best response rate with classical chemotherapy dacarbazine has ranged from 6 to $28 \%$, and survival has been absolutely disappointing. So it was a day to remember when the results of the BRIM III study were presented recently at the yearly American Society of Clinical Oncology (ASCO) meeting and published in the New England Journal of Medicine on the same day [1]. Six hundred and seventy five patients with Stage IIIc or Stage IV melanoma entered this study. All had been selected on the basis of the presence of an activating mutation of the BRAF gene (mutation V600). They were randomized to receive an anti-tyrosine kinase anti-Braf (vemurafenib) or dacarbazine. The results were very striking, with a reduction in the risk of death of $67 \%$ and a reduction in the risk of progression of $74 \%$ in the experimental arm when compared with the dacarbazine group.

This treatment stands as the first personalized treatment in melanoma based on a specific mutation, with a favorable impact on survival. According to Chapman, lead author (Memorial Sloan-Kettering Center in New York), "This is really a huge step toward personalized care in melanoma ... This is the first successful melanoma treatment tailored to patients who carry a specific gene mutation in their tumor, and could eventually become one of only two drugs available that improves overall survival in advanced cancers (melanoma)."

However, although the drug can be considered a new standard of treatment for patients with this gene mutation, several remarks come to mind with this study. First, this key trial opens a new era in drug development confirming the

J.-F. Morère $(\bowtie)$

Hôpital Avicenne, Université Paris XIII,

Paris-Nord,

Paris, France

e-mail: jean-francois.morere@avc.ap-hop-paris.fr
"Herceptin case." A new drug has to be developed with its specific predictive test. Second, several questions remain unsolved:

- How can we treat patients without Braf mutations (20 $50 \%$ of the patients)?

- Do we have to consider immune therapy with ipilumumab? This could be an option, even if a great tumor burden is not theoretically the ideal setting for immune therapy.

- Can we use vemurafenib in the adjuvant setting in the near future? The safety profile could be a concern. About 20 to $30 \%$ of patients developed an original side effect: low-grade skin cancers.

- Can we combine it? One candidate could be ipilumumab. One trial is already planned. Other candidates could be mitogen-activated protein/extracellular signal-regulated kinase kinase (MEK) inhibitors or multikinase inhibitors (RAF-MEK inhibitors). These inhibitors could be more efficient by overcoming the mechanisms of resistance and maybe by lowering the incidence of some side effects such as squamous carcinomas.

Despite the shadow of these questions, melanoma is no longer an orphan tumor, which is good news for our patients.

The journal Targeted Oncology is also the object of some recent good news: our impact factor has jumped to 2.054. As editor-in-chief, I would like to thank everyone who has contributed to the success of this journal.

\section{Reference}

1. Chapman PB, Hauschild A, Robert C et al (2011) Improved survival with vemurafenib in melanoma with BRAF V600E mutation. N Engl J Med 364:2507-2516 